\title{
Three-Dimensional Nanostructure of an Intact Microglia Cell
}

\author{
Giulia Bolasco ${ }^{1}$, Laetitia Weinhard ${ }^{1}$, Tom Boissonnet ${ }^{1}$, Ralph Neujahr ${ }^{2}$ and \\ Cornelius T. Gross ${ }^{1 *}$
}

${ }^{1}$ Epigenetics \& Neurobiology Unit, European Molecular Biology Laboratory (EMBL Rome), Monterotondo, Italy, ${ }^{2}$ Carl Zeiss Microscopy GmbH, ZEISS Group, Oberkochen, Germany

Keywords: microglia, TEM, CLEM, volumetric, ultrastructure, SBEM, segmentation

OPEN ACCESS

Edited by:

Jennifer Irene Luebke,

Boston University, United States

Reviewed by:

Giulia Poggi,

Friedrich Miescher Institute for Biomedical Research, Switzerland Marie-Eve Tremblay, Laval University, Canada

*Correspondence:

Cornelius T. Gross

cornelius.gross@embl.it

Received: 21 September 2018 Accepted: 20 November 2018 Published: 05 December 2018

Citation:

Bolasco G, Weinhard L, Boissonnet T, Neujahr $R$ and Gross CT (2018) Three-Dimensional Nanostructure of an Intact Microglia Cell.

Front. Neuroanat. 12:105

doi: 10.3389/fnana.2018.00105
Microglia are non-neuronal cells of the myeloid lineage that invade and take up long-term residence in the brain during development (Ginhoux et al., 2010) and are increasingly implicated in neuronal maturation, homeostasis, and pathology (Bessis et al., 2007; Paolicelli et al., 2011; Li et al., 2012; Aguzzi et al., 2013; Cunningham, 2013; Cunningham et al., 2013). Since the early twentieth century several methods for staining and visualizing microglia have been developed. Scientists in Ramón y Cajal's group (Achúcarro, 1913; Río-Hortega, 1919) pioneered these methods and their work led to the christening of microglia as the third element of the nervous system, distinct from astrocytes and neurons. More recently, a combination of imaging, genetic, and immunological tools has been used to visualize microglia in living brain (Davalos et al., 2005; Nimmerjahn et al., 2005). It was found that microglia are highly motile under resting conditions and rapidly respond to injuries (Kettenmann et al., 2011) suggesting a role for microglia in both brain homeostasis and pathology. Transmission Electron microscopy (TEM) has provided crucial complementary information on microglia morphology and physiology but until recently EM analyses have been limited to single or limited serial section studies (Tremblay et al., 2010, 2012; Paolicelli et al., 2011; Schafer et al., 2012; Sipe et al., 2016). TEM studies were successful in defining a set of morphological criteria for microglia: a polygonal nucleus with peripheral condensed chromatin, a relatively small cytoplasm with abundant presence of rough endoplasmic reticulum (RER), and a large volume of lysosomes and inclusions in the perikaryon. Recent advances in volumetric electron microscopy techniques allow for $3 \mathrm{D}$ reconstruction of large samples at nanometer-resolution, thus opening up new avenues for the understanding of cell biology and architecture in intact tissues. At the same time, correlative light and electron microscopy (CLEM) techniques have been extended to 3D brain samples to help navigate and identify critical molecular landmarks within large EM volumes (Briggman and Denk, 2006; Maco et al., 2013; Blazquez-Llorca et al., 2015; Bosch et al., 2015). Here we present the first volumetric ultrastructural reconstruction of a nearly complete mouse hippocampal microglia using serial block face scanning electron microscopy (SBEM). Imaging was performed on a mouse at early postnatal stage (P15) in CA1 stratum radiatum. Using CLEM we have ensured the inclusion of both large, small, and filopodial microglia processes. Segmentation of the dataset allowed us to carry out a comprehensive inventory of microglia cell structures, including vesicles, organelles, membrane protrusions, and processes. This study provides a reference that can serve as a data mining resource for investigating microglia cell biology.

\section{MATERIALS AND METHODS}

\section{Confocal Imaging and Laser Etching}

Thy1::EGFP; Cx3cr1::CreER; RC::LSL-tdTomato triple transgenic mice were bred, genotyped and tested at EMBL following protocols approved by the EMBL Animal Use Committee and the Italian Ministry of Health. Cre-mediated recombination was induced by a single injection of $98 \%$ 
1) Hippocampus sections (Vibratome- 60um)

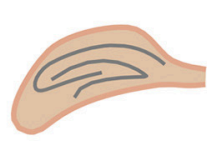

2) Confocal Imaging

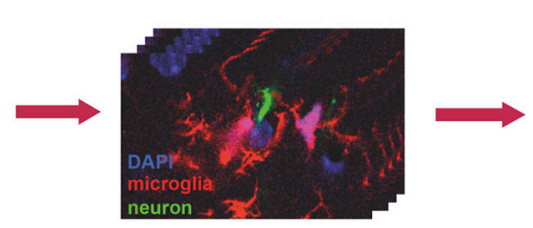

3) Tissue branding

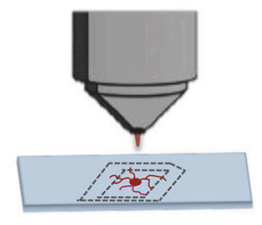

4) EM protocol:

- $2 \% \mathrm{OSO}_{4} / 1 \% \mathrm{~K}_{4} \mathrm{Fe}(\mathrm{CN})$

- THC treatment

- $2 \% \mathrm{OSO}_{4}$

- $1 \%$ Uranyl Acetate

- Lead Aspartate

- Dehydratation in Acetone

- Durcupan resin embedding

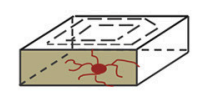

7) 3view Imaging

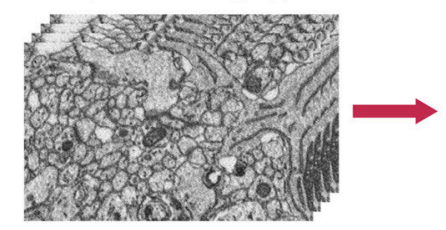

5) 3View SEM

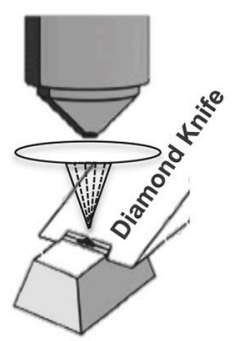

6) Low resolution CLEM

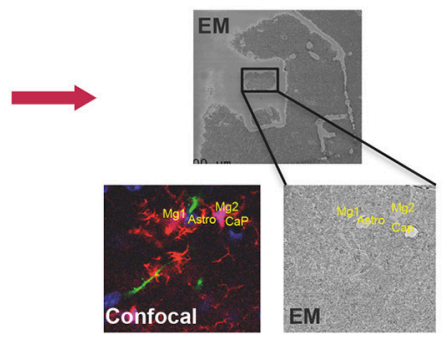

8) Segmentation (iMOD)

9) 3D Model (Blender)
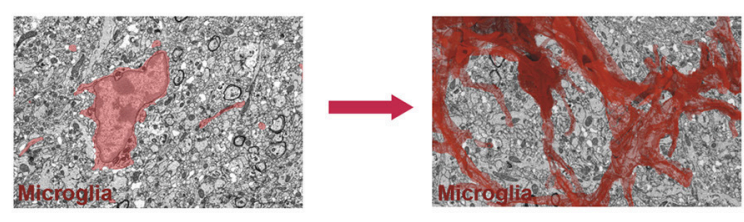

FIGURE 1 | Correlative light and electron microscopy workflow. Confocal imaging of microglia (tdTomato, red), cell nuclei (DAPI, blue), and sparsely labeled neurons (EGFP, green), in sections from mouse hippocampus was followed by tissue laser branding, sample preparation for electron microscopy and serial block face EM, manual segmentation (iMOD), and rendering (Blender) of the microglia, red.

Z-isomers hydroxy-tamoxifen diluted in corn oil at $10 \mathrm{mg} / \mathrm{mL}$ (Sigma, $1 \mathrm{mg}$ injected per $20 \mathrm{~g}$ of mouse weight) at P10. A male mouse was sacrificed at P15, perfused transcardially with PBS and fixed with Karnosky fixative [2\% (w/v) PFA, $2.5 \%(\mathrm{w} / \mathrm{v})$ Glutaraldehyde-TAAB] in $0.1 \mathrm{M}$ Phosphate Buffer (PB). After perfusion brains were dissected, trimmed in $x y$ around hippocampal areas and postfixed in $4 \%$ PFA in PB $0.1 \mathrm{M}$ overnight at $4^{\circ} \mathrm{C}$. Subsequently, $60 \mu \mathrm{m}$ thick vibratome (Leica Microsystems) coronal sections were cut and DAPI (Thermo Fisher) stained. Sections were mounted with 1\% Low Melting Agarose (Sigma) in PB 0.1M on glass bottom dishes with alphanumeric grid (Ibidi). Regions of interest (ROI) containing microglia were imaged in CA1 stratum radiatum at low and high magnification with TCS SP5 resonant scanner confocal microscope (Leica Microsystems) with a $63 \mathrm{x} / 1.2$ water immersion objective, at a pixel size of $48 \mathrm{~nm}$ and a step size of $300 \mathrm{~nm}$. Low magnification stacks containing the ROI were acquired in bright field, RFP, GFP, and DAPI channels, thus creating as a navigation map with internal fiducial markers (microglia, capillaries, and cell nuclei). A UV-diode laser operating at $405 \mathrm{~nm}$, a DPSS solid-state laser at $561 \mathrm{~nm}$, and an Argon laser at 488 were used as excitation sources. Subsequent to confocal imaging, the external side of the glass-bottom grid was wet with $50 \% \mathrm{EtOH}$, carefully detached from the plastic dish and placed onto Laser Capture Micro-dissector (LMD7000, Leica Microsystems) for laser etching of the ROI (Figure 1).

\section{Serial Block Face Scanning Electron Microscopy (SBEM)}

Laser branding sections were retrieved and stored in $0.5 \%$ PFA in $\mathrm{PB} 0.1 \mathrm{M}$ at $4^{\circ} \mathrm{C}$. Selected sections were shaped in asymmetric polygons around the ROI and coordinates were measured before proceeding for electron microscopy (EM) preparation (Figure 1). Briefly sections were washed in cold

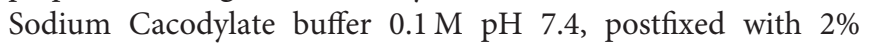
$\mathrm{OsO}_{4} / 1.5 \%$ Potassium Ferrocyanide for $1 \mathrm{~h}$ on ice, followed by a step with Thiocarbohydrazide for $20 \mathrm{~min}$ at RT and then a second step of $30 \mathrm{~min}$ in $2 \%$ aqueous $\mathrm{OsO}_{4}$ on ice. Samples were then rinsed carefully in water and stained "en block" first with $1 \%$ aqueous solution of Uranyl Acetate $\mathrm{ON}$ at $4^{\circ} \mathrm{C}$ and then with Lead Aspartate at $60^{\circ} \mathrm{C}$ for $30 \mathrm{~min}$. Subsequently, sections were dehydrated with increasing concentration of Acetone and infiltrated in Durcupan resin overnight followed by $2 \mathrm{~h}$ embedding step with fresh resin. Durcupan embedding was 
carried out in a flat orientation within a sandwich of ACLAR ${ }^{\circledR}$ $33 \mathrm{C}$ Films (Electron Microscopy Science) for $72 \mathrm{~h}$ at $60^{\circ} \mathrm{C}$. Upon embedding samples were trimmed using the coordinates to about $1 \mathrm{~mm}$ width to fit on the stab for 3View ultra-microtome (Gatan) and sputter coated with $5 \mathrm{~nm}$ Iridium (EMITECH). Samples were mounted into aluminum pin using double component Epoxy resin supplemented with Carbon anno-tubes to provide electrical conductivity. In order to have a broader field of view (FOV), data were acquired on 3view fitted into a Gemini 330VP (Zeiss, Germany) SEM (scanning EM) column. Images were acquired first at low resolution $\left(15^{*} 15 \mathrm{~nm} x y\right.$ pixel size $)$ and to reveal the etched marks from the laser micro-dissection, and correlate both vasculature and cell nuclei with the confocal imaging map. Upon pinpointing of the FOV containing the microglia of interest $\left(31.72 * 23.80^{*} 22.6 \mu \mathrm{m}\right)$ the imaging parameters were set to variable pressure with $5 \mathrm{~Pa}$ water vapor, $1.3 \mathrm{kV} \mathrm{HV}$ high current, dwell time $6 \mu \mathrm{s}$ at a pixel size of $5 \mathrm{~nm}$ and section thickness $25 \mathrm{~nm}$.

\section{Image Processing and Segmentation}

A single stack file containing the 3view/GeminiSEM generated dataset was aligned using ImageJ software (Rasband and
Bright, 1995, https://imagej.net/) with the plugin Linear Stack alignment with SIFT (Lowe, 2004, https://imagej.net/Linear_ Stack_Alignment_with_SIFT). To reduce dataset size and therefore further computation and memory usage, the stack was binned $2 \mathrm{X}$ in $x y$ axis, to obtain a final $x y$ resolution of $10 \times 10 \mathrm{~nm}$. At this resolution, it was still possible to identify the organelles and small processes. Microglia of interest was located based on its $x y z$ coordinates within the ROI and from fiducial objects correlated between EM and confocal datasets. Segmentation of microglia cell body was carried out manually using iMOD software (Kremer et al., 1996, http:// bio3d.colorado.edu/imod/) driven by a set of morphological criteria: the presence of abundant RER, the condensed chromatin at the periphery of the nucleus and the lack of mitochondria in the thin processes. A 3D model was then generated and correlated to the confocal dataset to confirm microglia identity. Systematic classification of organelles and vesicles trafficking within the cell was done by manual segmentation. Measurement of different color-code objects (Figure 2) was done by meshing the iMOD 3D model and exporting to the.obj format using built-in commands of iMOD (imodmesh.exe and imod2obj.exe). The generated mesh file was imported

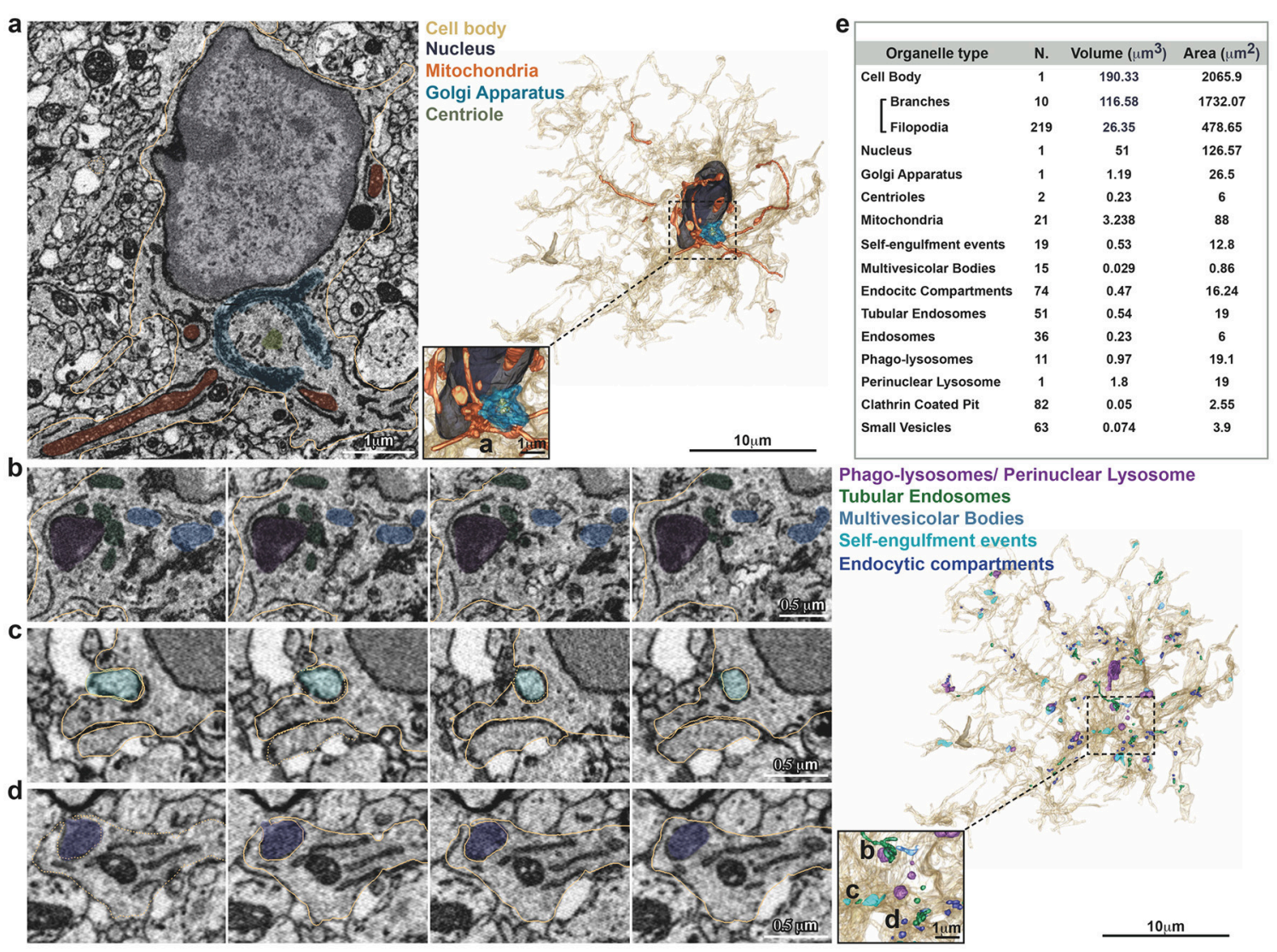

FIGURE 2 | Inventory of microglia structures. Serial Block Face EM images showing segmentation of selected color-coded objects within the microglia (a-d, gold). (a) Representative micrograph containing the microglia nucleus (dark gray), where also mitochondria (orange), Golgi apparatus (indigo), and centrioles (gray) are segmented (left). 3D surface rendering of peri-nuclear objects together with high magnification inset (right). (b-d) Representative series of consecutive micrographs showing the microglia: (b) multi-vesicular bodies (clear blue) and phago-lysosomes (purple); (c) self-engulfment of a branch originating from the same microglia (turquoise); (d) early endocytic compartment (dark blue). 3D surface rendering of intra-cellular vesicles, together with high magnification inset (right). (e) Total Volume and Area of segmented structures. 
in Blender (https://www.blender.org/) to create and render in $3 \mathrm{D}$ an animation of the full EM dataset combined with the 3D model (Supplementary Video 1). Moreover, NeuroMorph, plugin (Jorstad et al., 2018, https://github.com/NeuroMorphEPFL/NeuroMorph) was used to individually microglia's main branches and filopodia.

\section{DATABASE DESCRIPTION}

The Serial Block Face EM series of stack has been split in 2 separate datasets due to the $4 \mathrm{~GB}$ iMOD memory usage restrictions: -dataset1 - (sect 1 to 389) and -dataset2- (section 390904 ), respectively, with their $3 \mathrm{D}$ models set at $\pm 389 z$ offset. Each dataset collection is deposited at the Electron Microscopy Public Image Archive -EMPIAR- (Iudin et al., 2016) https:// www.ebi.ac.uk/pdbe/emdb/empiar/entry/10201, and should be opened together with the related model using $3 \mathrm{dmod}$ on iMOD software, previously downloaded at http://bio3d.colorado.edu/ $\mathrm{imod} /$. Both a Zap and an Information window will be opened. The Information window has the main controls for black and white contrast, and allows for opening the Model View window by clicking on the Image button. The ZaP (Zoom and Pan) window contains the stack with the different color-coded segmented objects, and allows, by using the toolbar, several controls such as zooming in/out and sliding through the sections. In the Model View window objects can be switched on and off using the Edit button and the model can be rotated or edited. Additional features can be found at http://bio3d.colorado.edu/ $\mathrm{imod} / \mathrm{doc} / 3 \mathrm{dmodguide.html.}$

Moreover a -Segmented.blend- file to visualize the stacks in $x, y, z$ orientations integrated with its 3D Model can be found in the database (above link). The file can be opened with Blender (https://www.blender.org/) and can be explored through upon installation of the provided

\section{REFERENCES}

Achúcarro, N. (1913). Notas sobre la estructura y funciones de la neuroglía y en particular de la neuroglía de la corteza cereral humana. Trab. Lab. Invest. Biol. Univ. 3, 1-31.

Aguzzi, A., Barres, B., and Bennett, M. L. (2013). Microglia: scapegoat, saboteur, or something else? Science 339, 156-161. doi: 10.1126/science.1227901

Bessis, A., Bechade, C., Bernard, D., and Roumier, A. (2007). Microglial control of neuronal death and synaptic properties. Glia 55, 233-238. doi: 10.1002/glia.20459

Blazquez-Llorca, L., Hummel, E., Zimmerman, H., Zou, C., Burgold, S., Rietdorf, J., et al., (2015). Correlation of two-photon in vivo imaging and FIB/SEM microscopy. J. Microsc. 259, 129-136. doi: 10.1111/jmi.12231

Bolasco, G., Weinhard, L., Boissonnet, T., Neujahr, R., and Gross, C. T. (2018). Three-dimensional nanostructure of an intact microglia cell. Biorxiv. [Preprint]. doi: 10.1101/388538

Bosch, C., Martinez, A., Masachs, N., Teixeira, C. M., Fernaud, I., Ulloa, F., et al. (2015). FIB/SEM technology and high-throughput 3D reconstruction of dendritic spines and synapses in GFP-labeled adult-generated neurons. Front. Neuroanat. 9:60. doi: 10.3389/fnana.2015.00060

Briggman, K. L., and Denk, W. (2006). Towards neural circuit reconstruction with volume electron microscopy techniques. Curr. Opin. Neurobiol. 16, 562-570. doi: $10.1016 /$ j.conb.2006.08.010 add-on -NeuroMorph_3D_Drawing.py- (see NeuroMorph documentation for help). Additional features are available with the complete NeuroMorph add-ons suite (https:/github.com/ NeuroMorph-EPFL/NeuroMorph). A more thorough protocol is available online (dx.doi.org/10.17504/protocols.io.u4eeyte), open to comments and suggestions.

To quickly present the dataset and show the rendering capabilities of Blender, a 3D animation -Blendervideo.avi- has also been stored in the repository. It shows the full SBEM dataset in the 3 orthogonal anisotropic planes meshed with the 3D model of few selected objects (microglia cell body-dark gray, nucleusblue, mitochondria-orange, and lyso-phagosomes-purple).

\section{AUTHOR CONTRIBUTIONS}

CG conceived the project. GB and LW set up the protocol and prepared the samples. GB segmented the full dataset and wrote the paper. RN ran the 3View SBEM system. TB processed and measured the $3 \mathrm{D}$ model and created the animation.

\section{FUNDING}

Funding has been provided by EMBL (CG, GB, LW, and TB) and ERC Advanced Grant COREFEAR (CG). A pre-print version of the manuscript has been published on bioRxiv (Bolasco et al., 2018).

\section{SUPPLEMENTARY MATERIAL}

The Supplementary Material for this article can be found online at: https://www.frontiersin.org/articles/10.3389/fnana. 2018.00105/full\#supplementary-material

Supplementary Video 1 | 3D Animation.

Cunningham, C. (2013). Microglia and neurodegeneration: The role of systemic inflammation. Glia 61, 71-90. doi: 10.1002/glia.22350

Cunningham, C. L., Martinez-Cerdeno, V., and Noctor, S. C. (2013). Microglia regulate the number of neural precursor cells in the developing cerebral cortex. J. Neurosci. 33, 4216-4233. doi: 10.1523/JNEUROSCI.3441-12. 2013

Davalos, D., Grutzendler, J., Yang, G., Kim, J. V., Zuo, Y., Jung, S., et al. (2005). ATP mediates rapid microglial response to local brain injury in vivo. Nat. Neurosci. 8, 752-758. doi: 10.1038/nn1472

Ginhoux, F., Greter, M., Leboeuf, M., Nandi, S., See, P., Gokhan, S., et al. (2010). Fate mapping analysis reveals that adult microglia derive from primitive macrophages. Science 330, 841-845. doi: 10.1126/science.1194637

Iudin, A. P. K., Korir, Salavert-Torres, J., Kleywegt, G. J., and Patwardhan, A. (2016). EMPIAR: a public archive for raw electron microscopy image data. Nat. Methods 13, 387-388. doi: 10.1038/nmeth.3806

Jorstad, A., Blanc, J., and Knott, G. (2018). NeuroMorph: a software toolset for 3D analysis of neurite morphology and connectivity. Front. Neuroanat. 12:59. doi: 10.3389/fnana.2018.00059

Kettenmann, H., Hanisch, U. K., Noda, M., and Verkhratsky, A. (2011). Physiology of microglia. Physiol. Rev. 91, 461-553. doi: 10.1152/physrev.00011.2010

Kremer, J. R., Mastronarde, D. N., and McIntosh, J. R. (1996). Computer visualization of three-dimensional image data using IMOD. J. Struct. Biol. 116, 71-76. doi: 10.1006/jsbi.1996.0013 
Li, Y., Du, X. F., Liu, C. S., Wen, Z. L., and Du, J. L. (2012). Reciprocal regulation between resting microglial dynamics and neuronal activity in vivo. Dev. Cell 23, 1189-1202. doi: 10.1016/j.devcel.2012.10.027

Lowe, D. G. (2004). Distinctive image features from scale-invariant keypoints. Int. J. Comput. Vis. 60, 91-110. doi: 10.1023/B:VISI.0000029664.99 615.94

Maco, B., Holtmaat, A., Cantoni, M., Kreshuk, A., Straehle, C. N., Hamprecht, F. A., et al. (2013). Correlative in vivo 2 photon and focused ion beam scanning electron microscopy of cortical neurons. PLOS ONE 8:e57405. doi: 10.1371/journal.pone.0057405

Nimmerjahn, A., Kirchhoff, F., and Helmchen, F. (2005). Resting microglial cells are highly dynamic surveillants of brain parenchyma in vivo. Science 308, 1314-1318. doi: 10.1126/science.1110647

Paolicelli, R. C., Bolasco, G., Pagani, F., Maggi, L., Scianni, M., Panzanelli, P., et al. (2011). Synaptic pruning by microglia is necessary for normal brain development. Science 333, 1456-1458. doi: 10.1126/science.1 202529

Rasband, W. S., and Bright, D. S. (1995). Nih image - a public domain imageprocessing program for the Macintosh. Microbeam Anal. 4, 137-149.

Río-Hortega, P. (1919). El "tercer elemento de los centros nerviosos". IV. Poder fagocitario y movilidad de la microglía. Bol. Soc. Esp. Biol. 8, 155-166.

Schafer, D. P., Lehrman, E. K., Kautzman, A. G., Koyama, R., Mardinly, A. R., Yamasaki, R., et al. (2012). Microglia sculpt postnatal neural circuits in an activity and complement-dependent manner. Neuron 74, 691-705. doi: 10.1016/j.neuron.2012.03.026
Sipe, G. O., Lowery, R. L., Tremblay, M. E., Kelly, E. A., Lamantia, C. E., and Majewska, A. K. (2016). Microglial P2Y12 is necessary for synaptic plasticity in mouse visual cortex. Nat. Commun. 7:10905. doi: 10.1038/ncomms10905

Tremblay, M. E., Lowery, R. L., and Majewska, A. K. (2010). Microglial Interactions with synapses are modulated by visual experience. PLoS Biol. 8:e1000527 . doi: 10.1371/journal.pbio.1000527

Tremblay, M. E., Zettel, M. L., Ison, J. R., Allen, P. D., and Majewska, A. K. (2012). Effects of aging and sensory loss on glial cells in mouse visual and auditory cortices. Glia 60, 541-558. doi: 10.1002/gli a.22287

Conflict of Interest Statement: RN was employed by Carl Zeiss Microscopy (Oberkochen, Germany).

The remaining authors declare that the research was conducted in the absence of any commercial or financial relationships that could be construed as a potential conflict of interest.

Copyright (c) 2018 Bolasco, Weinhard, Boissonnet, Neujahr and Gross. This is an open-access article distributed under the terms of the Creative Commons Attribution License (CC BY). The use, distribution or reproduction in other forums is permitted, provided the original author(s) and the copyright owner(s) are credited and that the original publication in this journal is cited, in accordance with accepted academic practice. No use, distribution or reproduction is permitted which does not comply with these terms. 